\title{
INDUCCIÓN DE MICRONÚCLEOS EN CÉLULAS MERISTEMÁTICAS DE LA RAÍZ DE Vicia faba TRATADAS CON DIFERENTES CONCENTRACIONES DE MARVEL®
}

\author{
Juana SÁNCHEZ ALARCÓN ${ }^{1,2,3}$, Monica PÉREZ-SÁNCHEZ ${ }^{1}$, José Luis GÓMEZ-OLIVARES ${ }^{2,4}$, \\ Rosa María LÓPEZ-DURÁN ${ }^{2,4}$, José Mariano Rigoberto MONTIEL-GONZÁLEZ ${ }^{1,3}$, \\ Rafael Alexander VALENCIA-SÁNCHEZ, Hipólito MUÑOZ-NAVA ${ }^{5}$, \\ Guillermo Alejandro PÉREZ FLORES ${ }^{1,3}$ y Rafael VALENCIA-QUINTANA ${ }^{1,2,3 *}$
}

${ }^{1}$ Laboratorio “Rafael Villalobos-Pietrini” de Toxicología Genómica y Química Ambiental, Facultad de Agrobiología, Universidad Autónoma de Tlaxcala

${ }_{3}^{2}$ Red Temática de Toxicología de Plaguicidas CONACyT-UANayarit

${ }^{3}$ Cuerpo Académico Ambiente y Genética UATLX-CA-223

${ }^{4}$ Laboratorio de Biomembranas, Departamento de Ciencias de la Salud, División de Ciencias Biológicas y de la Salud, Universidad Autónoma Metropolitana

${ }^{5}$ Facultad de Agrobiología, Universidad Autónoma de Tlaxcala Km. 10.5 Autopista San Martín - Tlaxcala, Ixtacuixtla de Mariano de Matamoros CP 90120, México

*Autor para correspondencia:prvq2004@yahoo.com.mx

(Recibido marzo 2017; aceptado septiembre 2017)

Palabras clave: Micronúcleos, atrazina, dicamba, índice mitótico

\section{RESUMEN}

El empleo de plaguicidas ha ido aumentando al paso del tiempo. A pesar de los beneficios aportados por los agroquímicos tanto en la agricultura como en el ámbito doméstico, muchos de ellos pueden representar peligros potenciales para la salud y el ambiente. En la actualidad es manifiesto el interés creciente en la determinación de biomarcadores que permitan la medición y estimación de una exposición activa y/o pasiva a contaminantes ambientales con capacidad tóxica, como los plaguicidas. Este estudio analiza el daño al ADN que causa el herbicida comercial Marvel ${ }^{\circledR}$ a través del ensayo de micronúcleos (MN), en células meristemáticas de la raíz de Vicia faba. Raíces de entre 2-3 cm fueron expuestas durante 4 horas, con 18 y 44 horas de recuperación a diferentes concentraciones de Marvel $\AA$, equivalentes a las siguientes combinaciones de atrazina/dicamba: 62.5/31.25; 125/62.5;250/125; 1000/400;2000/1000; 3000/1500 y 4000/2000 mg de ingrediente activo/L de agua destilada (mg I.A./L). Las células fueron expuestas a agua destilada, como testigo negativo y a dicromato de potasio $0.05 \%$, como testigo positivo, bajo las mismas condiciones experimentales. Con excepción de la concentración más baja [62.5/31.25 mg I.A./L], todas las concentraciones probadas presentaron un daño significativo $(\mathrm{p}<0.05)$. Con respecto al testigo negativo, el daño fue mayor a las 18 horas de recuperación, al paso de 44 horas, el daño se vio disminuido en todas las concentraciones probadas. El índice mitótico (IM) también se modificó, mostrándose una menor división dependiente de la concentración y tiempo de recuperación. Las concentraciones de 2000/1000; 3000/1500 y 4000/2000, mg I.A./L, produjeron un daño mayor que el encontrado en el testigo positivo. Los datos sugieren que el herbicida comercial Marvel ${ }^{\circledR}$ produce daño al ADN y es citotóxico en Vicia faba.

Key words: Micronucleus, atrazine, dicamba, mitotic index 


\begin{abstract}
The use of pesticides has been increasing over time. Despite the benefits provided by agrochemicals in agriculture as well as in domestic uses, many of them may pose potential health and environmental hazards. There is now increasing interest in the determination of biomarkers that allow the measurement and estimation of an active and / or passive exposure to environmental pollutants with toxic capacity, such as pesticides. This study analyzes the DNA damage caused by Marvel ${ }^{\circledR}$ a commercial herbicide through the micronucleus (MN) assay, in meristematic cells of the root of Vicia faba. Roots between 2-3 cm were exposed for 4 hours with 18 and 44 hours of recovery time at different concentrations of Marvel ${ }^{\circledR}$, equivalent to the following combinations of atrazine / dicamba: 62.5 / 31.25; 125 / 62.5; 250/125; 1000/500; 2000/1000; 3000/1500 and 4000/2000, $\mathrm{mg}$ of active ingredient / L of distilled water. Cells were exposed to distilled water as a negative control and potassium dichromate $(0.05 \%)$ as a positive control under the same experimental conditions. With the exception of the lowest concentration [62.5 / $31.25 \mathrm{mg}$ of active ingredient / $\mathrm{L}$ of distilled water], all concentrations tested showed significant damage $(\mathrm{p}<0.05)$, with respect to the negative control, the damage was greater at 18 hours of recovery, and at 44 hours the damage was decreased at all concentrations tested. The mitotic index (MI) also was affected showing a smaller division depending on concentration and recovery times. The concentrations of 2000/1000; 3000/1500 and 4000/2000, mg of active ingredient / L of distilled water produced greater damage than that found in the positive control. This leads to the conclusion that the commercial herbicide Marvel ${ }^{\circledR}$ causes DNA damage and is cytotoxic in Vicia faba.
\end{abstract}

\section{INTRODUCCIÓN}

Los plaguicidas se emplean comúnmente en todas las zonas agrícolas del mundo, con el propósito de proteger los cultivos contra organismos nocivos que merman la producción (Rasgele et al. 2014). Sin embargo, a pesar de los beneficios asociados con su uso, muchos de éstos representan riesgos potenciales para la salud pública y el ambiente debido a sus posibles efectos citotóxicos / genotóxicos (Valencia-Quintana et al. 2016a).

Muchos plaguicidas, tal como se comercializan y utilizan, son formulaciones de dos componentes principales, los ingredientes "activos" (I.A.) y los ingredientes "inertes". Estos últimos pueden tener actividad biológica propia, y pueden representar un riesgo potencial para causar efectos tóxicos en la salud humana (Cox y Surgan 2006). En el estado de Tlaxcala, dos de los herbicidas más frecuentemente utilizados son el dicamba [un herbicida auxínico, perteneciente a la familia de sales del ácido benzoico] y la atrazina [de la familia de triazinas], que solos o en combinación son empleados para controlar las malas hierbas en cultivos de maíz (Reynoso et al. 2015).

El producto comercial Marvel ${ }^{\circledR}$ es un herbicida post-emergente que combina la acción sistémica y de contacto para el control de la maleza, en los cultivos de maíz, sorgo, caña de azúcar y pastizales. Marvel ${ }^{\circledR}$ es una formulación comercial a base de dicamba [ácido 3,6-dicloro-2- metoxi benzoico al 11.25\% (equivalente a $132 \mathrm{~g}$ de I.A./L)] y atrazina [6-cloro$\mathrm{N}$-etil-N-isopropil- 1,3,5-triazina-2,4-diamina al $21.48 \%$ (equivalente a $252 \mathrm{~g}$ de I.A./L)] (Cuadro I), como I.A., e indica un $67.27 \%$ de ingredientes inertes (humectante, dispersante, espesante, antiespumante y disolvente) (Cuadro II) (Agromich 2017).

La atrazina es un herbicida selectivo que se aplica al suelo, se absorbe por las raíces o las hojas de las hierbas y se aplica antes o después de que germine la maleza. Controla la aparición de malezas en cultivos, principalmente de maíz, sorgo, caña de azúcar y trigo. En México, la importación de productos que contienen atrazina como I.A. ha aumentado de $189.35 \mathrm{t}$ en 2003 a $287.8 \mathrm{t}$ en 2012 , provenientes de Estados Unidos de América, China, India, Israel e Italia (SIAVI 2012).

La exposición a la atrazina puede darse en el ambiente laboral, ya sea por vía inhalatoria o dérmica. Puede producir reacciones de irritación, así como alteraciones en las funciones de algunos órganos, problemas de reproducción, alteración en los niveles hormonales (disruptor endocrino), así como defectos en el nacimiento en seres humanos (ATSDR, 2003, Brender y Weyer 2016). En animales se ha encontrado que la atrazina ocasiona alteraciones neuronales, en el desarrollo, daño al hígado, riñón y corazón, así 
CUADRO I. PROPIEDADES FISICOQUÍMICAS DE LOS PLAGUICIDAS EVALUADOS

\begin{tabular}{|c|c|c|}
\hline Parámetro & Atrazina & Dicamba \\
\hline Estructura química & & \\
\hline Formula química & $\mathrm{C}_{8} \mathrm{H}_{14} \mathrm{ClN}_{5}$ & $\mathrm{C}_{8} \mathrm{H}_{6} \mathrm{Cl}_{2} \mathrm{O}_{3}$ \\
\hline Peso molecular (fw) & $215.68 \mathrm{~g} / \mathrm{mol}$ & $221.04 \mathrm{~g} / \mathrm{mol}$ \\
\hline Puntos de fusión (mp) & $173-177^{\circ} \mathrm{C}$ & 114 y $116^{\circ} \mathrm{C}$ \\
\hline Presión de vapor (vp) & $3.8 \times 10^{-5} \mathrm{~Pa}$ & $3.4 \times 10^{-5} \mathrm{Hg}$ a $25^{\circ} \mathrm{C}$ \\
\hline Solubilidad (SW) & $35 \mathrm{mg} / \mathrm{L}$ en agua a $25^{\circ} \mathrm{C}$ & $6500 \mathrm{mg} / \mathrm{L}$ en agua a $25^{\circ} \mathrm{C}$ \\
\hline Vida media (por biodegradación) & 10 días (5 a 5824 días) & (4 a 555 días) \\
\hline Constante de la Ley de Henry (KH) & $\begin{array}{l}3.04 \times 10^{-9} \text { atm m } \\
\text { (Hansen et al. } 2013 \text { ) }\end{array}$ & $\begin{array}{c}2 \times 10^{-9} \text { atm-cu m/mol } \\
(\text { Syngenta } 2015)\end{array}$ \\
\hline
\end{tabular}

\section{CUADRO II. FICHA TÉCNICA MARVEL $®$}

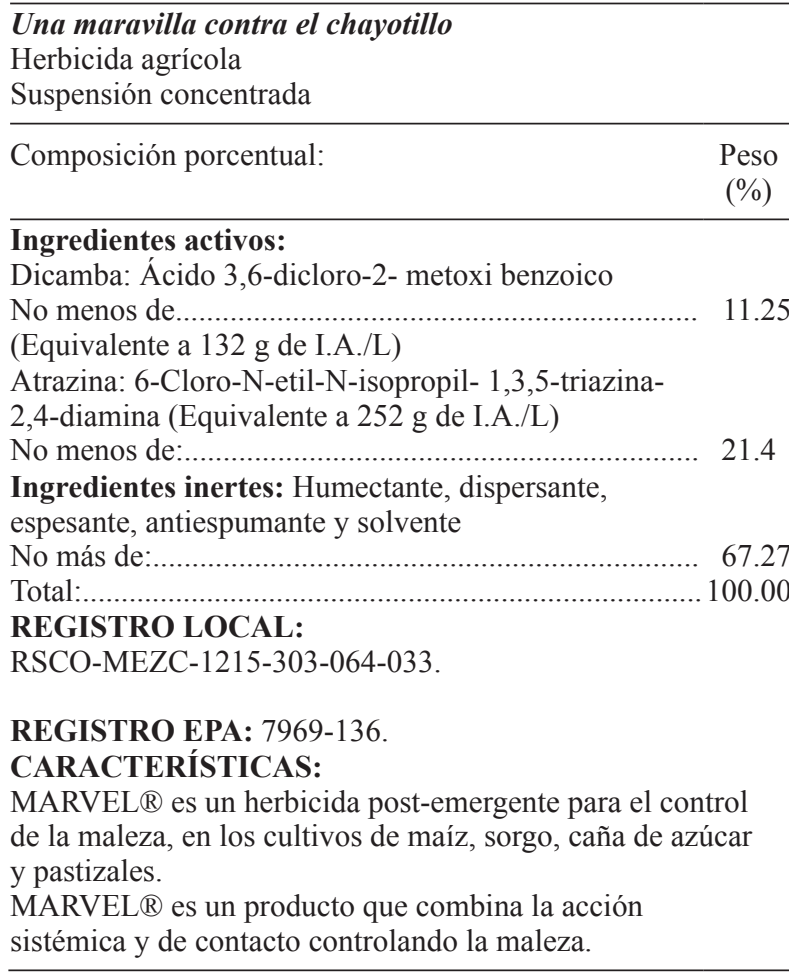

como cáncer (Gammon et al. 2005, Hornstein 2007). La atrazina se considera ligeramente tóxica para la vida acuática (Graymore et al. 2001) y es un inductor de hermafroditismo en ranas (Gammon et al. 2005). Asimismo, la Agencia para la Protección Ambiental de los Estados Unidos (USEPA) determinó que existe evidencia de carcinogenicidad del herbicida en animales, más no en seres humanos (USEPA, 2003). Estudios epidemiológicos han investigado la posibilidad de que la atrazina pueda tener efectos en humanos, algunos afirman que la exposición a ésta resulta en un riesgo elevado de cáncer de próstata, sin embargo estos son cuestionables (Gammon et al. 2005, Jowa y Howd 2011, Goodman et al. 2014).

Dicamba es un herbicida sistémico selectivo, absorbido por las hojas y raíces, actúa como un regulador del crecimiento-auxina causando crecimiento incontrolado. Se utiliza para el control de malezas de hoja ancha anuales, perennes y especies arbustivas, en cereales, maíz, sorgo, caña de azúcar, espárragos, pastos, pastizales y tierras no cultivadas (Syngenta, 2015).

En humanos, dicamba presenta una toxicidad aguda baja, puede tener efectos irritantes o corrosivos en piel y ojos, la USEPA lo ha identificado como capaz de alterar el desarrollo. No es tóxico para abejas, anfibios y crustáceos. Es ligeramente tóxico para peces y moderadamente tóxico para el zooplancton. Su toxicidad en aves y mamíferos es muy baja. Sin embargo, existen algunas evidencias que muestran una reducción ligera en la supervivencia de los huevos de gallina, así como efectos neurológicos en ratas, perros y gallinas. A pesar de ello, no se considera un riesgo significativo para la vida silvestre (Mergel 2011a).

Compuestos agrícolas han sido detectados en recursos acuáticos, como el agua potable, ríos y aguas subterráneas. Uno de los más frecuentemente encontrados es la atrazina (Brender y Weyer 2016). $\mathrm{Su}$ prevalencia en el agua potable, junto con el flujo de informes a veces conflictivos sobre los efectos ambientales y en la salud humana, ha generado una gran controversia en torno a su seguridad (Mergel 2011b). Por su parte, dicamba puede presentar alta movilidad en el suelo y puede fácilmente contaminar el agua (Mergel 2011a). 
Por ello es relevante detectar los posibles efectos negativos en diferentes organismos.

Entre los daños causados por los agentes químicos a las poblaciones ocupacional o no ocupacionalmente expuestas, los efectos genotóxicos son de los más peligrosos, debido a que pueden causar diferentes problemas de salud y también, afectar a generaciones futuras. Esta preocupación ha llevado al desarrollo de distintos ensayos de genotoxicidad en diversos organismos. Entre los más de 200 bioensayos conocidos en la literatura, las plantas superiores son consideradas como excelentes indicadores de efectos citogenéticos y mutagénicos de contaminantes ambientales. Estos bioensayos son confiables y muy sensibles para el monitoreo y la evaluación de agentes genotóxicos (Valencia-Quintana et al. 2013, 2016b). Vicia faba ha sido considerada como un sistema de prueba ideal para evaluar genotoxicidad y ha sido incluida en el programa Gene-Tox (Ma 1999).

Hoy en día los ensayos citogenéticos más comúnmente utilizados son las frecuencias de micronúcleos (MN) y de aberraciones cromosómicas (AC). Los $\mathrm{MN}$ han sido considerados por muchos autores como uno de los biomarcadores más sencillos y efectivos para analizar los efectos genotóxicos inducidos por agentes químicos (Valencia-Quintana et al. 2013). La formación de MN puede ser resultado de daños directos a los cromosomas o alteraciones al huso y al aparato mitótico (Çavuşoğlu et al. 2012).

Diversos estudios han proporcionado pruebas de que los plaguicidas poseen propiedades genotóxicas en diferentes bioensayos. Sin embargo, la información sobre los efectos genotóxicos de algunos herbicidas sigue siendo limitada y controversial (Reynoso et al. 2015). Por otra parte, la creciente evidencia científica ha demostrado que la evaluación de formulaciones comerciales de plaguicidas ha sido muy escasa. Por tal motivo y debido a la carencia de información sobre los efectos genéticos de Marvel ${ }^{\circledR}$, y a su uso asiduo en la agricultura, en este estudio se utilizó la prueba de MN en células meristemáticas de la raíz de Vicia faba con el propósito de evaluar la capacidad genotóxica de este herbicida.

\section{METODOLOGÍA}

Para verificar el efecto genotóxico de Marvel ${ }^{\circledR}$ sobre las células meristemáticas de las raíces de Vicia faba (minor), se aplicó la prueba de MN. Semillas de Vicia faba fueron lavadas y embebidas durante 24 horas en agua del grifo, posteriormente se dejaron germinar por 48-72 horas entre dos capas de algodón humedecido. Una vez que transcurrió este tiempo y las raíces alcanzaron entre $2-3 \mathrm{~cm}$, se expusieron durante 4 horas a diferentes concentraciones de Marvel ${ }^{\circledR}$, equivalentes a las siguientes combinaciones de atrazina/dicamba: 62.5/31.25; 125/62.5;250/125; $1000 / 400 ; 2000 / 1000 ; 3000 / 1500$ y $4000 / 2000$, mg I.A./L.

Los tratamientos se realizaron a $20^{\circ} \mathrm{C}$ en la oscuridad. Una vez transcurridos, se cortaron dos milímetros de las puntas de las raíces de Vicia faba, se tiñeron con Feulgen y se hicieron permanentes usando la técnica de hielo seco, deshidratadas con dos cambios de butanol (Gómez-Arroyo y VillalobosPietrini 1995, Valencia-Quintana et al. 2016a, b) y montadas con Entellan ${ }^{\circledR}$.

Los testigos negativo y positivo se manejaron bajo las mismas condiciones experimentales, pero las raíces de Vicia faba se sumergieron en agua destilada para el primer caso y para el segundo en dicromato de potasio $(0.05 \%)$, el cual ha demostrado su capacidad para inducir daño genético en este sistema de prueba.

Las laminillas fueron manejadas en ciego para evitar prejuicios por parte del observador. El análisis estadístico de los resultados se llevó a cabo mediante la prueba de $\chi^{2}$. Además, con el propósito de medir el grado de relación entre las diferentes concentraciones de Marvel ${ }^{\circledR}$ y la frecuencia de $\mathrm{MN}$, así como con el IM, se obtuvo el coeficiente de correlación de Pearson.

\section{RESULTADOS}

Las frecuencias de MN y los efectos sobre el IM inducidos en las células meristemáticas de la raíz de Vicia faba son presentados en las figuras $\mathbf{1}$ y $\mathbf{2}$, respectivamente.

Al exponer directamente las raíces de Vicia faba a las diferentes concentraciones del herbicida Marvel ${ }^{\circledR}$, se observa que este plaguicida es capaz de incrementar la frecuencia de MN, tanto a las 18 como a las 44 horas de recuperación (Figura 1). $\mathrm{La}$ frecuencia de MN presenta un comportamiento dosis-respuesta. Sin embargo, a las 44 horas de recuperación se observa que las frecuencias de MN disminuyen de forma significativa al compararse con las frecuencias obtenidas a las 18 horas de recuperación, lo que puede representar la capacidad de recuperación de las células meristemáticas de la raíz de Vicia faba al daño inducido.

Los efectos de Marvel ${ }^{\circledR}$ sobre el IM de las células meristemáticas de la raíz de Vicia faba, son presentados en la figura 2. En el grupo testigo el IM 


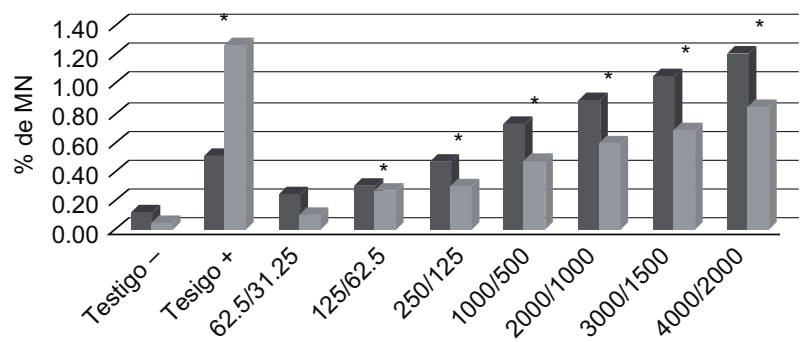

Concentración de Marvel ${ }^{\circledR}$ [Atrazina/Dicamba] (mg I.A./L)

18 horas de recuperación 44 horas de recuperación

Fig. 1. Frecuencia de micronúcleos $(\mathrm{MN})$ inducidos por diferentes concentraciones de ingrediente activo (I.A.) de Marvel ${ }^{\circledR}$ después de 4 horas de tratamiento con 18 y 44 horas de recuperación en células meristemáticas de la raíz de Vicia faba.

*Diferencia significativa $\mathrm{p}<0.05$ prueba de $\chi^{2}$, con respecto a su testigo negativo

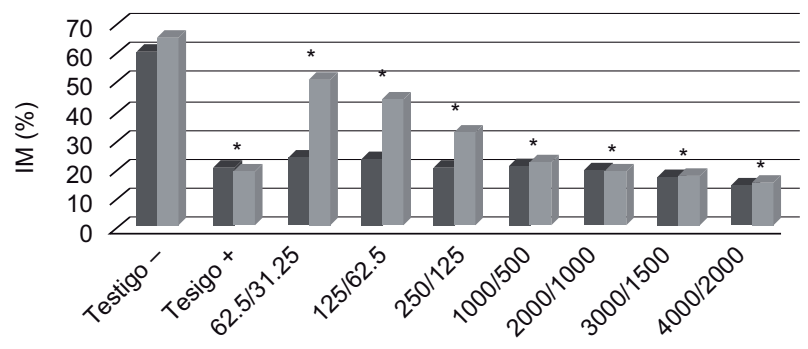

Concentración de Marve ${ }^{\circledR}[$ Atrazina/Dicamba] (mg I.A./L)

- 18 horas de recuperación 44 horas de recuperación

Fig. 2. Efectos sobre el índice mitótico (IM) inducidos por diferentes concentraciones de ingrediente activo (I.A.) de Marvel ${ }^{\circledR}$ después de 4 horas de tratamiento con 18 y 44 horas de recuperación en células meristemáticas de la raíz de Vicia faba.

* Diferencia significativa $\mathrm{p}<0.05$ prueba de $\chi^{2}$, con respecto a su testigo negativo

fue aproximadamente de un $60 \%$. Al comparar los grupos tratados y testigo se observa una disminución del IM con 18 horas de recuperación. Los grupos tratados presentan un comportamiento asintótico ya que tuvieron un valor casi uniforme a lo largo de las diferentes concentraciones del herbicida probadas y siendo estadísticamente similar al valor inducido por el dicromato de potasio $(0.05 \%)$ que fue utilizado como testigo positivo. Por otra parte, a las 44 horas de recuperación se observa una incremento del IM en las concentraciones más bajas probadas de Marvel ${ }^{\circledR}$ [atrazina/dicamba] (62.5/31.25 - 250/125 mg I.A./L), al compararse con los valores obtenidos con 18 horas de recuperación, aunque los valores siguen siendo menores que el observado en el testigo negativo y va disminuyendo conforme aumenta la concentración del herbicida a la cual fueron expuestas. A partir de 1000/500 mg I.A./L y hasta la concentración más alta probada, los valores del IM presentan valores similares a los encontrados a las 18 horas con las mismas concentraciones y similares a los valores encontrados con el testigo positivo.

\section{DISCUSIÓN}

La demanda de mayores rendimientos de los cultivos y la reducción de las pérdidas posteriores a las cosechas, alienta a los productores agrícolas a hacer uso extensivo de los plaguicidas (Zhang et al. 2011). Sin embargo, éstos son uno de los contaminantes más peligrosos para la salud humana y el ambiente (Costa et al. 2006, Feffstrup et al. 2010, Lee et al. 2012). Así, la detección y/o evaluación de su genotoxicidad son cruciales porque las personas expuestas a estos productos pueden verse afectadas negativamente en su estado de salud (Rasgele et al. 2014).

La atrazina es uno de los herbicidas más empleados a nivel mundial, está restringido en los Estados Unidos y ha sido prohibido en varios países de la Comunidad Europea. Sin embargo, en México su uso no está regulado y es de amplia aplicación en zonas de cultivo (Hansen et al. 2013). El nivel de genotoxicidad provocado por la atrazina y sus formulaciones comerciales ha sido controversial, resultados positivos y negativos han sido encontrados en diferentes sistemas de prueba (Brusick 1994, Ribas et al. 1995, Kligerman et al. 2000, Brand y Mueller 2002, Želježić et al. 2006, Song et al. 2009, Srivastava y Mishra 2009, Nwani et al. 2011, Campos-Pereira et al. 2012). En el caso de dicamba y sus formulaciones, han sido reportados como capaces de inducir daño genético (Filkowski et al. 2003, González et al. 2007, 2009, Cenkci et al. 2010).

Diversos estudios sobre los efectos genotóxicos y/o citotóxicos obtenidos con principios activos y con formulaciones comerciales de diversos plaguicidas indican que estas últimas pueden contener xenobióticos que pueden modificar la toxicidad intrínseca de los I.A., de ahí la importancia de evaluar no sólo el I.A. sino también los productos comerciales, que son en última instancia los que representan la realidad de exposición en los trabajadores agrícolas (Brand y Mueller 2002, Želježić et al. 2006, González et al. 2007, 2009, Costa el al. 2009, Molinari et al. 2009, 
Padula et al. 2012, Nikoloff et al. 2013, 2014, Yadav 2013, Pérez et al. 2014, Pérez-Iglesias et al. 2014, Ruiz de Arcaute et al. 2014, Rasgele et al. 2014, Ramos-Chávez et al. 2015, Çayır et al. 2016, Valencia-Quintana et al. 2016a, b).

Las observaciones realizadas en este trabajo después del tratamiento de las células meristemáticas de Vicia faba con el herbicida Marvel® sugieren que éste efectivamente causa daño genético evidenciado a través de la presencia de MN, así como la disminución de la división celular, disminuyendo el IM de manera significativa (Figuras 1 y 2).

El cálculo del coeficiente de correlación de Pearson indica que existe correlación positiva entre la concentración de Marvel ${ }^{\circledR}$ y la frecuencia de MN. Los datos soportan esta hipótesis tanto a las $18(\mathrm{r}=0.9435)$, como a las 44 horas de recuperación $(\mathrm{r}=0.9485)$. La prueba de $\chi^{2}$, demostró que a partir de $125 / 62.5 \mathrm{mg}$ I.A./L, existe diferencia significativa en la frecuencia de MN en comparación con el testigo negativo.

Al comparar los efectos inducidos a las 18 y 44 horas de recuperación, la prueba de $\chi^{2}$, indica que, con un $99 \%$ de confianza, existe diferencia significativa entre ambos tiempos $(\mathrm{p}<0.0001)$. Esto es un indicio de la capacidad de recuperación de las células meristemáticas de la raíz de Vicia faba al daño inducido, ya que éste presenta una disminución a las 44 horas al compararse con el inducido con 18 horas de recuperación. Además se encontró que la frecuencia de MN sigue un comportamiento logarítmico con un R2 mayor a 0.95

De igual manera, el coeficiente de correlación de Pearson indica una correlación negativa entre la concentración de Marvel ${ }^{\circledR}$ y el IM. Además, las efectos inducidos por el tiempo de recuperación se pueden apreciar claramente a bajas concentraciones de Marvel ${ }^{\circledR}$ ya que a 44 horas se observa una incremento con respecto al inducido con 18 horas de recuperación. Sin embargo, a partir de 1000/500 mg I.A./L, los IM son muy similares en ambos tiempos de recuperación, lo cual parece indicar que una concentración alta genera daños de tal mesura que las células no pueden recuperarse.

El efecto de altas concentraciones de Marvel ${ }^{\circledR}$ en las células meristemáticas de la raíz de Vicia faba es mayor en el IM que en la frecuencia de MN ya que el tiempo de recuperación tiene efectos diferenciales sobre ambos biomarcadores. Aunque la recuperación del daño genético es manifiesta a las 44 horas, en el caso del IM solo es hasta la concentración de 250/125 mg I.A./L, mientras que para la frecuencia de MN se observa en todas las concentraciones probadas.
Además, la correlación entre concentración de Marvel ${ }^{\circledR}$ y la frecuencia de $\mathrm{MN}$ sigue un comportamiento logarítmico, lo que implica que el mayor daño se induce con concentraciones de Marvel ${ }^{\circledR}$ bajas. Al aumentar la concentración el daño se incrementa pero no en igual magnitud. No obstante, las pruebas realizadas en este estudio indican que Marvel ${ }^{\circledR}$ causa daño genotóxico y citotóxico, pero hacen falta más estudios para comprender mejor el alcance del efecto que este agente puede ocasionar.

Las concentraciones de I.A. utilizadas en el presente estudio, pueden considerarse ambientalmente no relevantes, quizá solo observadas cuando ocurren eventos específicos (v. gr. la aplicación directa). Sin embargo, no se puede descartar la posibilidad de que los trabajadores agrícolas pudieran estar expuestos accidentalmente a estas concentraciones elevadas (Valencia-Quintana et al. 2016a, b). Para el maíz, la concentración de Marvel ${ }^{\circledR}$ recomendada por el fabricante es hasta de $1850 \mathrm{mg} / \mathrm{L}$ de agua (Cuadro III). Las concentraciones usadas en el presente estudio estuvieron alrededor del valor recomendado, probando cuatro concentraciones por debajo y tres por arriba. Algunos agricultores reportan que utilizan este producto a una concentración aproximada de $375 \mathrm{mg} / \mathrm{L}$. Sin embargo, como puede observarse ni la concentración recomendada por el fabricante, ni la reportada por los agricultores es segura, dado que éstas son capaces de inducir daños cito- y genotóxicos de acuerdo con los resultados del presente estudio.

Son pocos los estudios que se han realizado para evaluar el potencial genotóxico y/o citotóxico de diferentes formulaciones comerciales de plaguicidas mediante diversos sistemas de prueba (Koller et al. 2012, Valencia-Quintana et al. 2016a, b). Aneuploidía y poliploidía han sido inducidas por atrazina en $\mathrm{Al}$ lium cepa y Vicia faba (Srivastava y Mishra, 2009), al igual que fragmentos cromosómicos (Bolle et al. 2004), lo cual podría explicar la presencia de $\mathrm{MN}$ en las células meristemáticas de la raíz de Vicia faba en este trabajo.

La formación de $\mathrm{MN}$ puede ser resultado de daños directos a los cromosomas o alteraciones al huso y al aparato mitótico (Çavuşoğlu et al. 2012). Los MN pueden originarse como resultado de la exclusión de fragmentos acéntricos o de cromosomas con centrómero inactivo, fuera de la envoltura nuclear durante el término de la mitosis. Las anafases y telofases multipolares conducen a la formación de células multinucleadas en interfase y algunos cromosomas "retardados" o con centrómero inactivado pueden potencialmente agruparse y formar también MN. Además, los MN también pueden originarse de la 
CUADRO III. MARVEL $®$, RECOMENDACIONES DE USO CULTIVOS, PLAGAS, DOSIS

\begin{tabular}{|c|c|c|c|}
\hline Cultivo & Nombre común & Nombre científico & Dosis (L/ha) \\
\hline Maíz & $\begin{array}{l}\text { Chayotillo } \\
\text { Quelite } \\
\text { Aceitilla } \\
\text { Mostaza } \\
\text { Quelite cenizo } \\
\text { Cardo blanco } \\
\text { Girasol silvestre } \\
\text { Rosilla chica }\end{array}$ & $\begin{array}{l}\text { Sicyos angulata } \\
\text { Amaranthus sp } \\
\text { Bidens frondosa } \\
\text { Brassica campestris } \\
\text { Chenopodium album } \\
\text { Cirsium sp } \\
\text { Helianthus annuus } \\
\text { Gallinsoga hispida }\end{array}$ & $\begin{array}{c}2.0 \text { a } 3.0 \\
\text { Aplique en post-emergencia a la maleza cuando ésta } \\
\text { tenga entre } 5 \text { y } 15 \mathrm{~cm} \text { de altura. } \\
\text { Respecto al cultivo la aplicación en post-emergencia } \\
\text { se puede realizar en forma total desde que el maíz } \\
\text { nace hasta que tenga } 30 \mathrm{~cm} \text { de altura, si es mayor } \\
\text { realice aplicaciones dirigidas hasta } 10 \text { días antes del } \\
\text { espigamiento. }\end{array}$ \\
\hline Sorgo & $\begin{array}{l}\text { Chayotillo } \\
\text { Quelite } \\
\text { Aceitilla } \\
\text { Mostaza } \\
\text { Quelite cenizo } \\
\text { Cardo blanco } \\
\text { Girasol silvestre } \\
\text { Rosilla chica }\end{array}$ & $\begin{array}{l}\text { Sicyos sp } \\
\text { Amaranthus sp } \\
\text { Bidens frondosa } \\
\text { Brassica campestris } \\
\text { Chenopodium album } \\
\text { Cirsium sp } \\
\text { Helianthus annuus } \\
\text { Gallinsoga hispida }\end{array}$ & $\begin{array}{l}1.5 \text { a } 2.0 \\
\text { Aplique en post-emergencia a la maleza, cuando ésta } \\
\text { tenga una altura entre } 5 \text { y } 10 \mathrm{~cm} \text {. } \\
\text { Para el control de chayotillo utilice la dosis máxima. } \\
\text { Aplique en post-emergencia al cultivo en forma total. }\end{array}$ \\
\hline \multirow[t]{2}{*}{$\begin{array}{l}\text { Caña de } \\
\text { azúcar }\end{array}$} & $\begin{array}{l}\text { Andan o gigantón } \\
\text { Girasol } \\
\text { Flor amarilla } \\
\text { Quelite } \\
\text { Campanilla } \\
\text { Quelite cenizo }\end{array}$ & $\begin{array}{l}\text { Tithonia tubaeformis } \\
\text { Helianthus annuus } \\
\text { Melampodium perfoliatum } \\
\text { Amaranthus sp } \\
\text { Ipomoea purpurea } \\
\text { Chenopodium } \\
\text { album }\end{array}$ & $\begin{array}{c}1.0 \\
\text { Aplique en post-emergencia a la maleza cuando ésta } \\
\text { tenga entre } 5 \text { y } 15 \mathrm{~cm} \text { de altura. }\end{array}$ \\
\hline & Chayotillo & Sicyos angulata & $\begin{array}{c}2.0 \\
\text { Hacer las aplicaciones cuando la maleza tenga de } 10 \text { a } \\
15 \mathrm{~cm} \text { y antes de que la caña cierre el surco. }\end{array}$ \\
\hline $\begin{array}{l}\text { Pastizales } \\
\text { (Maleza } \\
\text { anual) }\end{array}$ & $\begin{array}{l}\text { Hierba Martín } \\
\text { Abrojo } \\
\text { Amargosa } \\
\text { Bledo } \\
\text { Quelite cenizo } \\
\text { Cadillo } \\
\text { Mostaza } \\
\text { Ortiga } \\
\text { Salvia } \\
\text { Verdolaga }\end{array}$ & $\begin{array}{l}\text { Hiptys sp } \\
\text { Achyrantes indica } \\
\text { Parthenium hysterophorus } \\
\text { Amaranthus sp } \\
\text { Chenopodium album } \\
\text { Xanthium sp } \\
\text { Brassica campestris } \\
\text { Urtica dioica } \\
\text { Loeselia ciliata } \\
\text { Portulaca oleracea }\end{array}$ & $\begin{array}{l}1.0 \text { a } 1.5 \text { en } 200 \mathrm{~L} \text { de agua. Asperje la maleza a punto } \\
\text { de goteo. }\end{array}$ \\
\hline $\begin{array}{l}\text { Pastizales } \\
\text { (Maleza } \\
\text { perene) }\end{array}$ & $\begin{array}{l}\text { Cardo } \\
\text { Gloria de la mañana } \\
\text { Diente de león } \\
\text { Golondrina } \\
\text { Lengua de vaca }\end{array}$ & $\begin{array}{l}\text { Cirsium sp } \\
\text { Ipomoea dissecta } \\
\text { Taraxacum officinale } \\
\text { Euphorbia sp } \\
\text { Rumex crispus }\end{array}$ & $\begin{array}{c}1.5 \text { a } 2.0 \\
\text { en } 200 \mathrm{~L} \text { de agua. } \\
\text { Asperje la maleza a punto de goteo. }\end{array}$ \\
\hline
\end{tabular}

eliminación de ADN excedente del núcleo principal en un intento por restaurar las condiciones normales de ploidía (Valencia-Quintana et al. 2013). En este estudio se tuvo la presencia de MN; por el tamaño que presentan se puede pensar que la mayoría de ellos fueron originados por fragmentos de cromosomas.

Diversos estudios han presentado que los radicales libres pueden causar inestabilidad genómica al provocar desórdenes en el citoesqueleto, desbalance en el metabolismo energético y daño al ADN que puede llevar a la inducción de alteraciones cromosómicas. Los efectos encontrados en la presente investigación pueden ser atribuidos al estrés oxidante inducido por Marvel®. Como soporte de esta hipótesis está el hecho de que algunos plaguicidas tienen la capacidad de alterar el potencial redox en células vegetales (Jain y Bhalla-Sarin 2001, Miteva et al. 2005, 2010, Siddiqui et al. 2012), o de inhibir enzimas que participan en 
la defensa contra especies nocivas de oxígeno, como la superóxido dismutasa, la catalasa y la glutatión transferasa (Rannug y Rannug 1984).

Tanto la reducción como el incremento en el IM son indicadores importantes en el monitoreo de la contaminación ambiental, especialmente para la evaluación de contaminantes que presentan potencial tóxico y citotóxico. Así, diversos estudios han usado la evaluación del IM para detectar citotoxicidad y la mayoría ha presentado resultados positivos (SmakaKinel et al. 1996; Bolle et al. 2004, Fernandes et al. 2007, Türkoğlu, 2007). La inducción de anormalidades mitóticas en las células meristemáticas de las raíces de las plantas puede causar un decremento del IM (Kovalchuk et al. 1998; Bushra et al. 2002). Es por ello que en el presente trabajo se tomó en cuenta este parámetro, ya que como se ha citado antes, es un indicador recomendable, deduciendo que Marvel ${ }^{\circledR}$ no solo es un agente genotóxico sino también tiene un efecto tóxico que interfiere con la progresión del ciclo celular.

Estudios citogenéticos han evidenciado que la mayoría de los plaguicidas afectan la división celular, este efecto ha sido reportado para la atrazina en $\mathrm{Al}$ lium cepa (Bolle et al. 2004) y Vicia faba (Srivastava y Mishra, 2009), así mismo, en este trabajo, una vez más se evidencia su capacidad de daño por la exposición de Vicia faba a la fórmula comercial Marvel®.

El efecto sobre el proceso de mitosis podría deberse a su acción inhibidora sobre la síntesis de ADN, ARN y proteínas y / o fibras del huso. Además, se sabe que algunos plaguicidas tienen la capacidad de bloquear la progresión en el ciclo celular en fase $\mathrm{G}_{2}-\mathrm{M}$ inhibiendo la activación de CDK1 / ciclina (Granby y Vahl 2001, Marc et al. 2002, 2004a, b, Ismail et al. 2009, Siddiqui et al. 2012).

Estudios recientes demostraron que dicamba se debe considerar como un agente que daña el ADN. La inducción de ICH y la alteración tanto en la progresión del ciclo celular y los IM se ha encontrado en linfocitos humanos in vitro y células de ovario de hámster Chino (CHO-K1) (González et al. 2006; 2007). Además, se informó que el herbicida induce roturas de hebra de ADN reveladas por el ensayo cometa (González et al. 2007), así como MN en células de CHO-K1 (González et al. 2011). Por último, también se reportaron resultados positivos en la aplicación del ensayo de MN en Tradescantia (Mohammed y Ma 1999).

Por lo antes citado se demuestra que tanto la atrazina como dicamba inducen daño al ADN de forma independiente. En el presente estudio la combinación de éstos en el producto comercial
Marvel ${ }^{\circledR}$ fue capaz de inducir daño al ADN por la presencia de $\mathrm{MN}$ y una disminución en la división celular dependiendo las concentraciones. Reynoso et al. (2015), realizaron el primer experimento con la formulación comercial utilizada en este trabajo. A través del ensayo cometa visualizan el daño genotóxico después de haber sometido los núcleos de los meristemos apicales de hojas de 119 variedades de maíz dulce de México y Sudamérica con 1000-2000 y 2000-4000 ppm de dicamba-atrazina. El daño encontrado en ambas concentraciones probadas fue significativo al compararse con el testigo negativo. Sin embargo, al ser comparadas entre ellas no presentaron diferencias estadísticamente significativas, sucediendo lo contrario en el presente estudio, ya que en éste se presentó una relación dosis-respuesta en todas las concentraciones probadas, aun siendo concentraciones similares en ambos estudios. Lo anterior puede representar una diferencia en la sensibilidad de los biomarcadores y/o bioensayos empleados. No obstante, en ambos estudios se deduce que la mezcla es genotóxica.

Por último, los resultados presentados son consistentes con la recomendación de que la evaluación de los efectos genotóxicos del I.A. de un plaguicida no es suficiente, sino que es indispensable evaluar los diferentes productos comerciales disponibles en el mercado. Los efectos perjudiciales del / los excipiente (s) presentes en la formulación comercial no deben ser descartados ni subestimados. Los componentes de las formulaciones de los plaguicidas usados como polvos humectables consisten usualmente en el vehículo, arcillas minerales, agentes tensioactivos y otros ingredientes tales como estabilizadores y tintoreros. La presencia de agentes tensioactivos podría aumentar la actividad genotóxica del I.A., favoreciendo la cantidad de metabolitos activos en objetivos celulares críticos (Bernardes et al. 2015).

\section{CONCLUSIONES}

El presente estudio utilizó el ensayo de MN para demostrar que una formulación comercial de dicamba-atrazina (Marvel ${ }^{\circledR}$ ) puede ser genotóxica y citotóxica para células meristemáticas de la raíz de Vicia faba. Los resultados presentados indican que éste producto comercial puede significativamente dañar el ADN de los organismos expuestos y provocar daño citotóxico al interferir en su división celular, bajo las condiciones experimentales empleadas. Lo anterior sugiere la necesidad de tomar las medidas necesarias al usar este producto en áreas agrícolas. 
Los sistemas vegetales pueden servir como excelentes monitores para la detección de sustancias químicas ambientales que pueden plantear un riesgo genético.

En conjunto, los hallazgos sugieren la importancia de nuevos estudios sobre este tipo de plaguicidas con el fin de lograr un conocimiento completo sobre su genotoxicidad. Se necesitan estudios futuros in vivo o in vitro para elucidar su mecanismo de acción.

\section{AGRADECIMIENTOS}

Los autores agradecen el financiamiento recibido del CONACyT a través del proyecto de la Red Temática de Plaguicidas con número 280045.

\section{REFERENCIAS}

Agromich (2017). Marvel®. Ficha técnica. Agroquímicos de Michoacán SA de CV http://www.agromich.com/ imagenes/productos/marvel/43.pdf. Consultado el 18 de febrero de 2017.

ATSDR (2003). Reseña toxicológica de la atrazina. Agencia para Sustancias Tóxicas y Registro de Enfermedades. Departamento de Salud y Servicios Humanos de los EUA. www.atsdr.cdc.gov/es. Consultada el 10/10/2016.

Bernardes M.F.F., Pazin M., Pereira L.C. y Dorta D.J. (2015). Impact of pesticides on environmental and human health. En: Toxicology Studies-Cells, Drugs and Environment (Andreazza C y Scola G Eds.). InTech, Croacia, pp. 195-233. DOI: 10.5772/59710

Bolle P., Mastrangelo S., Tucci P. y Evandri M.G. (2004). Clastogenicity of atrazine assessed with the Allium cepa test. Environ Mol Mutagen. 43, 137-141. DOI: $10.1002 / \mathrm{em} .20007$

Brand R.M. y Mueller C. (2002). Transdermal penetration of atrazine, alachlor, and trifluralin: effect of formulation. Toxicol. Sci. 68, 18-23.

DOI: $10.1093 /$ toxsci/68.1.18

Brender J.D. y Weyer P.J. (2016). Agricultural compounds in water and birth defects. Curr. Environ Health Rep. 3, 144-152. DOI: 10.1007/s40572-016-0085-0.

Brusick D.J. (1994). An assessment of the genetic toxicity of atrazine: relevance to human health and environmental effects. Mutat. Res. 317, 133-144. DOI: 10.1016/0165-1110(94)90021-3

Bushra A., Abdul Farah M., Niamat Alı M. y Ahmad N. (2002). Clastogenicity of pentachlorophenol, 2, 4-D and butachlor evaluated by Allium root tip test. Mutat. Res. 514, 105-113.

DOI: $10.1016 / \mathrm{S} 1383-5718(01) 00327-8$
Campos-Pereira F.D., Oliveira C.A., Pigoso A.A., SilvaZacarin E.C., Barbieri R., Spatti E.F., Marin-Morales M.A. y Severi-Aguiar G.D. (2012). Early cytotoxic and genotoxic effects of atrazine on Wistar rat liver: a morphological, immunohistochemical, biochemical, and molecular study. Ecotoxicol. Environ. Saf. 78, 170-177. DOI: 10.1016/j.ecoenv.2011.11.020

Çavuşoğlu K., Kaya A., Yilmaz F. y Yalçin E. (2012). Effects of cypermethrin on Allium cepa. Environ. Toxicol. 27, 583-589. DOI: 10.1002/tox.20681

Çayır A., Coşkun M. y Coşkun M. (2016). Genotoxicity of commercial fungicide Cabrio Plus on human cell. Cytotechnology 68, 1697-1704.

DOI: 10.1007/s10616-015-9919-0.

Cenkci S., Yildiz M., Ciğerci I.H., Bozdağ A., Terzi H. y Terzi E.S. (2010). Evaluation of 2,4-D and Dicamba genotoxicity in bean seedlings using comet and RAPD assays. Ecotoxicol. Environ. Saf. 73, 1558-1564.

DOI: 10.1016/j.ecoenv.2010.07.033

Costa C., Teixeira J.P., Silva S., Roma-Torres J., Coelho P., Gaspar J., Alves M., Laffon B., Rueff J. y Mayan O. (2006). Cytogenetic and molecular biomonitoring of a Portuguese population exposed to pesticides. Mutagenesis 21, 343-350. DOI:10.1093/mutage/gel039

Costa C., Silvari V., Melchini A., Catania S., Heffron J.J., Trovato A. y De Pasquale R. (2009). Genotoxicity of imidacloprid in relation to metabolic activation and composition of the commercial product. Mutat. Res. 672, 40-44.

DOI: 10.1016/j.mrgentox.2008.09. 018

Cox C. y Surgan M. (2006). Unidentified inert ingredients in pesticides: implications for human and environmental health. Environ. Health Perspect. 114, 1803-1806. DOI: $10.1289 /$ ehp. 9374

Feffstrup T.K., Larsen J.C. y Meyer O. (2010). Risk assessment of mixtures of pesticides. Current approaches and future strategies Regulat. Toxicol. Pharmacol. 56, 174-192. DOI: 10.1016/j.yrtph.2009.09.013

Fernandes T.C.C., Mazzeo D.E.C. y Marin-Morales M.A. (2007). Mechanism of micronuclei formation in polyploidizated cells of Allium cepa exposed to trifluralin herbicide. Pest. Biochem. Physiol. 88, 252-259.

DOI: 10.1016/j.pestbp.2006.12.003

Filkowski J., Besplug J., Burke P., Kovalchuk I. y Kovalchuk O. (2003). Genotoxicity of 2,4-D and dicamba revealed by transgenic Arabidopsis thaliana plants harboring recombination and point mutation markers. Mutat. Res. 542, 23-32.

DOI: 10.1016/j.mrgentox. 2003.07.008

Gammon D.W., Aldous C.N., Carr W.C. Jr, Sanborn J.R. y Pfeifer K.F. (2005). A risk assessment of atrazine use in California: human health and ecological aspects. Pest. Manag. Sci. 61, 331-355. DOI: 10.1002/ps. 1000 
Gómez-Arroyo S. y Villalobos-Pietrini R. (1995). Chromosomal aberrations and sister chromatid exchanges in Vicia faba as genetic monitors of environmental pollutants. En: Biomonitors and biomarkers as indicator of environmental change (Butterworth FM, Corkum LD, Guzmán-Rincón J, Eds.). Plenum Press, Nueva York. pp. 95-113

González N.V., Soloneski S. y Larramendy M.L. (2006). Genotoxicity analysis of the phenoxy herbicide dicamba in mammalian cells in vitro. Toxicol. In Vitro 20, 1481-1487. DOI: 10.1016/j.tiv.2006.05.001

González N.V., Soloneski S. y Larramendy M.L. (2007). The chlorophenoxy herbicide dicamba and its commercial formulation banvel induce genotoxicity in Chinese hamster ovary cells. Mutat. Res. 634, 60-68. DOI:10.1016/j.mrgentox.2007.06.001

González N.V., Soloneski V. y Larramendy M.L. (2009). Dicamba-induced genotoxicity in chinese hamster ovary $(\mathrm{CHO})$ cells is prevented by vitamin E. J. Hazard. Mater. 163, 337-343.

DOI: 10.1016/j.jhazmat.2008.06.097

González L., Sanderson B.J.S. y Kirsch-Volders M. (2011). Adaptation of the in vitro MN assay for the genotoxicity assessment of nanomaterials. Mutagenesis 26, 185-191. DOI: 10.1093/ mutage/geq088

Goodman M., Mandel J.S., DeSesso J.M. y Scialli A.R. (2014). Atrazine and pregnancy outcomes: A systematic review of epidemiologic evidence. Birth Defects Res. B Dev. Reprod. Toxicol. 101, 215-236.

DOI: 10.1002/bdrb.21101

Granby K. y Vahl M. (2001) Investigation of herbicide glyphosate and plant growth regulators chlormequat and mepiquat in cereals produced in Denmark. Food Addit. Contam. 18, 898-905.

DOI: $10.1080 / 02652030119594$

Graymore M., Stagnitti F. y Allinson G. (2001). Impacts of atrazine in aquatic ecosystems. Environ. Int. 26, 483-495. DOI: $10.1016 / \mathrm{S} 0160-4120(01) 00031-9$

Hansen A., Treviño-Quintanilla I., Márquez-Pacheco H., Villada-Canela M., González-Márquez L.C., GuillénGarcés R.A. y Hernández-Antonio A. (2013). Atrazina un herbicida polémico. Rev. Int. Contam. Ambie. 29 (Número especial sobre plaguicidas), 65-84. http:// www.redalyc.org/articulo.oa? $\mathrm{id}=37028958004$

Hornstein D.T. (2007). The Road Also Taken: Lessons from organic agriculture for market- and risk-based regulation. Duke L. J. 56, 1541-1580.

DOI: $10.2307 / 40040581$

Ismail C., Atilla Y., Yusuf T. y Levent O. (2009). Glyphosate reduced seed and leaf concentrations of calcium, manganese, magnesium and iron in non-glyphosate resistant soybean. Eur. J. Agro. 31, 114-119.

DOI: 10.1016/j.eja.2009.07.001
Jain M. y Bhalla-Sarin N. (2001). Glyphosate-induced increase in glutathione S-transferase activity and glutathione content in groundnut (Arachis hypogaea L.) Pest. Biochem. Physiol. 69, 143-152.

DOI: $10.1006 /$ pest.2000.2535

Jowa L. y Howd R. (2011). Should atrazine and related chlorotriazines be considered carcinogenic for human health risk assessment? J. Environ. Sci. Health C Environ. Carcinog. Ecotoxicol. Rev. 29, 91-144. DOI: 10.1080/10590501.2011.577681.

Kovalchuk O., Kovalchuk I., Arkhıpov A. Telyuk P., Hohn B. y Kovalchuk L. (1998). The Allium cepa chromosome aberration test reliably measures genotoxicity of soils of inhabited areas in the Ukraine contaminated by the Chernobyl accident. Mutat. Res. 415, 47-57. DOI: 10.1016/S1383-5718(98)00053-9

Kligerman A., Doerr D., Tennant A.H. y Peng B. (2000). Cytogenetic studies of three triazine herbicides II. In vivo micronucleus studies in mouse bone marrow. Mutat. Res. 471, 107-112. DOI: $10.1016 / \mathrm{S} 1383-5718(00) 00124-8$

Koller V.J., Fürhacker M., Nersesyan A., Mišík M., Eisenbauer M. y Knasmueller S. (2012). Cytotoxic and DNA-damaging properties of glyphosate and Roundup in human-derived buccal epithelial cells. Arch. Toxicol. 86, 805-813. DOI: 10.1007/s00204-012-0804-8

Lee W.J., Cha E.S., Park J., Ko Y., Kim H.J. y Kim J. (2012). Incidence of acute occupational pesticide poisoning among male farmers in South Korea. Am. J. Ind. Med. 55, 799-807 DOI: 10.1002/ajim.22024

Ma T.-H. (1999). The international program on plant bioassays and the report of the follow-up study after the hands-on workshop in China. Mutat. Res. 426, 103106. DOI: 10.1016/S0027-5107(99)00049-4

Marc J., Mulner-Lorillon O., Boulben S., Hureau D., Durand G. y Belle R. (2002). Pesticide roundup provokes cell division dysfunction at the level of CDK1/Cyclin B activation. Chem. Res. Toxicol. 15, 326-331. DOI: $10.1021 / \mathrm{tx} 015543 \mathrm{~g}$

Marc J., Mulner-Lorillon O. y Belle R. (2004a). Glyphosate-based pesticides affect cell cycle regulation. Biol. Cell 96, 245-249. DOI: 10.1016/j.biolcel.2003.11.010

Marc J., Belle R., Julia M., Patrick C. y Lorillon O.M. (2004b). Formulated glyphosate activates the DNAresponse check point of the cell cycle leading to the prevention of G2/M transition. Toxicol. Sci. 82, 436442. DOI: $10.1093 /$ toxsci/kfh 281

Mergel M. (2011a). Dicamba. Toxipedia. Conecting science and people. http://www. toxipedia.org/display/ toxipedia/Dicamba. Consultada el 28 de julio de 2017.

Mergel M. (2011b). Atrazine. Toxipedia. Conecting science and people. http://www. toxipedia.org/display/ toxipedia/Atrazine. Consultada el 28 de julio de 2017. 
Miteva L., Tsoneva J., Ivanov S. y Alexieva V. (2005). Alterations of the content of hydrogen peroxide and malondialdehyde and the activity of some antioxidant enzymes in the roots and leaves of pea and wheat plants exposed to glyphosate. Compt. Rend. Acad. Bulg. Sci., 58, 733-738. Cote INIST : 2359, 35400013801056.0170

Miteva L.P.E., Ivanov S.V. y Alexieva V.S. (2010) Alterations in glutathione pool and some related enzymes in leaves and roots of pea plants treated with herbicide glyphosate. Russ J. Plant Physiol. 57, 131-136. DOI: $10.1134 / \mathrm{S} 1021443710010188$

Mohammed K.B. y Ma T.H. (1999). Tradescantia-micronucleus and -stamen hair mutation assays on genotoxicity of the gaseous and liquid forms of pesticides. Mutat. Res. 426, 193-199.

DOI: 10.1016/S0027-5107(99)00067-6

Molinari G., Soloneski S., Reigosa M.A. y Larramendy M.L. (2009). In vitro genotoxic and cytotoxic effects of ivermectin and its formulation ivomec $₫$ on Chinese hamster ovary (CHOK1) cells. J. Hazard Mater. 165, 1074-1082. DOI: 10.1016/j.jhazmat.2008.10.083

Nikoloff N., Escobar L., Soloneski S. y Larramendy M.L. (2013). Comparative study of cytotoxic and genotoxic effects induced by herbicide S-metolachlor and its commercial formulation Twin Pack Gold ${ }^{\circledR}$ in human hepatoma (HepG2) cells. Food Chem. Toxicol. 62, 777-781. DOI: $10.1016 /$ j.fct.2013.10.015

Nikoloff N., Natale G.S., Marino D., Soloneski S. y Larramendy M.L. (2014). Flurochloridone-based herbicides induced genotoxicity effects on Rhinella arenarum tadpoles (Anura: Bufonidae). Ecotoxicol. Environ. Saf. 100, 275-281. DOI: 10.1016/j.ecoenv.2013. 10.021

Nwani C.D., Nagpure N.S., Kumar R., Kushwaha B., Kumar P. y Lakra W.S. (2011). Mutagenic and genotoxic assessment of atrazine-based herbicide to freshwater fish Channa punctatus (Bloch) using micronucleus test and single cell gel electrophoresis. Environ. Toxicol. Pharmacol. 31, 314-322.

DOI 10.1016/j.etap.2010.12.001

Padula G., Ponzinibbio M.V., Picco S. y Seoane A. (2012). Assessment of the adverse effects of the acaricide amitraz: in vitro evaluation of genotoxicity. Toxicol. Mech. Methods 22, 657-661. DOI: $10.3109 / 15376516.2012 .666683$

Pérez D.J., Lukaszewicz G., Menone M.L., V Amé M. y Camadro E.L. (2014). Genetic and biochemical biomarkers in the macrophyte Bidens laevis L. exposed to a commercial formulation of endosulfan. Environ. Toxicol. 29, 1063-1071. DOI: 10.1002/tox.21836

Pérez-Iglesias J.M., Ruiz de Arcaute C., Nikoloff N., Dury L., Soloneski S., Natale G.S. y Larramendy M.L. (2014). The genotoxic effects of the imidacloprid-based insecticide formulation Glacoxan Imida on Montevideo tree frog Hypsiboas pulchellus tadpoles (Anura, Hylidae). Ecotoxicol. Environ. Saf. 104, 120-126. DOI: 10.1016/j.ecoenv. 2014.03.002

Ramos-Chávez L.A., Sordo M., Calderon-Aranda E., Castañeda-Saucedo E., Ostrosky-Wegman P. y MorenoGodinez M.E. (2015). A permethrin/allethrin mixture induces genotoxicity and cytotoxicity in human peripheral blood lymphocytes. J. Toxicol. Environ. Health A 78, 7-14. DOI: 10.1080/15287394.2015.956025

Rannug A. y Rannug U. (1984). Enzyme inhibition as a possible mechanism of the mutagenicity of dithiocarbamic acid derivatives in Salmonella typhimurium. Chem. Biol. Interact. 49, 329-340.

DOI: 10.1016/0009-2797(84)90106-6

Rasgele P.G., Muranli F.D. y Kekeçoğlu M. (2014). Assessment of the genotoxicity of propineb in mice bone marrow cells using micronucleus assay. Cytol. Genet. 48, 233-237.

DOI:10.3103/S0095452714040045

Reynoso M.S., Alvarez C.M., De la Cruz L., Escoto M.D. y Sánchez J.J. (2015). Evaluation of the genotoxic activity of dicamba and atrazine herbicides in several Mexican and South American varieties of sweetcorn (Zea mays L.). Gen. Mol. Res. 14, 16585-16593.

DOI: $10.4238 / 2015$

Ribas G., Frenzilli G., Barale R. y Marcos R. (1995). Herbicide-induced DNA damage in human lymphocytes evaluated by the single-cell gel electrophoresis (SCGE) assay. Mutat. Res. 344, 41-54.

DOI: 10.1016/0165-1218(95)90037-3

Ruiz de Arcaute C., Soloneski S. y Larramendy M.L. (2014) Evaluation of the genotoxicity of a herbicide formulation containing 3,6-dichloro-2-metoxybenzoic acid (dicamba) in circulating blood cells of the tropical fish Cnesterodon decemmaculatus. Mutat. Res. 773, 1-8. DOI: $10.1016 /$ j.mrgentox.2014.08.001

SIAVI (2012). Secretaría de Economía, México. Sistema de Información Arancelaria Vía Internet. [en línea]. http://www.siavi.economia.gob.mx/. (Último acceso agosto 21, 2015).

Siddiqui S., Meghvansi M.K. y Khan S.S. (2012). Glyphosate, alachor and maleic hydrazide have genotoxic effect on Trigonella foenum-graecum L. Bull. Environ. Contam. Toxicol. 88, 659-665. DOI: 10.1007/s00128-012-0570-6.

Smaka-Kınel V., Stegnar P., Lovka M. y Toman J. (1996). The evaluation of waste, surface and ground water quality using the Allium test procedure. Mutat. Res. 368, 171-179.

DOI: 10.1016/S0165-1218(96)90059-2

Song Y., Zhu L.S., Wang J., Wang J.H., Liu W. y Xie H. (2009). DNA damage and effects on antioxidative 
enzymes in earthworm (Eisenia foetida) induced by atrazine. Soil Biol. Biochem. 41, 905-909.

DOI: 10.1016/j.soilbio.2008.09.009

Srivastava K. y Mishra K.K. (2009). Cytogenetic effects of cormmercially formulated atrazine on the somatic cells of Allium cepa and Vicia faba. Pest. Biochem. Physiol. 93, 8-12. DOI: 10.1016/j.pestbp.2008.08.001

Syngenta (2015). Marvel. http://www.syngenta.com.mx/ Data/Sites/1/agroquimicos_productos/herbicidas / marvel/ marvel_info.pdf. Consultada el 19/11/2015

Türkoğlu S. (2007). Genotoxicity of five food preservatives tested on root tips of Allium cepa L. Mutat. Res. 626, 4-14.

DOI: $10.1016 /$ j.mrgentox.2006.07.006

USEPA (2003). Interim Reregistration eligibility decision for Atrazine. United States Environmental Protection Agency. Washington, D.C. 285 pp. www.pmra-arla. gc.ca. Consultada el 11/12/2016.

Valencia-Quintana R., Sánchez Alarcón J., Gómez-Arroyo S., Cortés Eslava J., Waliszewski S.M., Fernández S., Villalobos-Pietrini R. (2013). Genotoxicidad de plaguicidas en sistemas vegetales. Rev. Int. Contam. Ambie. 29 (Número especial sobre plaguicidas), 133157. http://www.redalyc.org/html/370/37028958008/ index.html

Valencia-Quintana R., Gómez-Arroyo S., SánchezAlarcón J., Milić M., Gómez-Olivares J.L., Waliszewski S.M., Cortés-Eslava J., Villalobos-Pietrini R. y Calderón-Segura M.E. (2016a). Assessment of genotoxicity of Lannate-90® and its plant and animal metabolites in human lymphocyte cultures. Arh. Hig. Rada. Toksikol. 67, 116-125.

DOI: 10.1515/aiht-2016-67-2763.

Valencia-Quintana R., Gómez-Arroyo S., SánchezAlarcón J., Milić M., Gómez-Olivares J.L., Waliszewski S.M., Cortés-Eslava J., Villalobos-Pietrini R. y Calderón-Segura M.E. (2016b). Genotoxic effects of the carbamate insecticide Pirimor-50® in Vicia faba root tip meristems and human lymphocyte culture after direct application and treatment with its metabolic extracts. Arh. Hig. Rada. Toksikol. 67, 266-276. DOI: 10.1515/aiht-2016-67-2809.

Yadav S.S. (2013). Toxic and genotoxic effects of Roundup on tadpoles of the Indian skittering frog (Euflictis cyanophlyctis) in the presence and absence of predator stress. Aquat. Toxicol. 132-133, 1-8. DOI: 10.1016/j.aquatox.2013.01.016

Želježić D., Garaj-Vrhovac V. y Perković P. (2006). Evaluation of DNA damage induced by atrazine and atrazinebased herbicide in human lymphocytes in vitro using a comet and DNA diffusion assay. Toxicol. In Vitro 20, 923-935. DOI: 10.1016/ j.tiv.2006.01.017

Zhang W., Jiang F. y Feng Ou J. (2011). Global pesticide consumption and pollution: with China as a focus. Proc. Int. Acad. Ecol. Environ. Sci. 1, 125-144. http://www.iaees.org/ publications/journals/piaees/ articles/2011-1\%282\%29/Global-pesticide-consumption-pollution.pdf 